\title{
REPERTÓRIO BOTÂNICO DA "PITOMBEIRA" (Talisia esculenta (A. ST.-HIL.) RADLK. - SAPINDACEAE)
}

\section{Germano GUARIM NETO', Santina Rodrigues SANTANA ${ }^{2}$, Josefa Valdete Bezerra da SILVA ${ }^{3}$}

\begin{abstract}
RESUMO - Os autores apresentam os resultados obtidos com o estudo de Talisia esculenta (A. St.-Hil.) Radlk. (Sapindaceae), denominada principalmente de "pitomba" ou "pitombeira", envolvendo aspectos botânicos referentes à morfologia, taxonomia, distribuição geográfica e respectivos usos. A espécie é bastante difundida no Brasil, sendo que nas regiões norte e nordeste o seu uso é intenso.
\end{abstract}

Palavras-chave: Talisia esculenta, Sapindaceae, morfologia, taxonomia, fitogeografia, importância econômica.

\section{Botanical repertorium of the "pitombeira" (Talisia esculenta (A. St.-Hil.) Radlk. - Sapindaceae}

\begin{abstract}
The authors present results on research with Talisia esculenta (A. St.-Hil.) Radlk. (Sapindaceae) commonly know as "pitomba" or "pitombeira" with botanical aspects concerning the morphology, taxonomy, geographical distribution and uses. The species is widely distributed in Brazil and is more frequently used in the north and northeast regions of the country.
\end{abstract}

Key-words: Talisia esculenta, Sapindaceae, morphology, taxonomy, phytogeography, uses.

\section{INTRODUÇÃO}

A família Sapindaceae Jussieu, importante componente da divisão Magnoliophyta, classe Magnoliopsida (cronquist, 1981) ou das populares angiospermas dicotiledôneas, ocorre principalmente nas regiões tropicais (lawrence, 1951), com uma distribuição em áreas bastante diversificadas, cujos gêneros e espécies compõem tanto a configuração vegetacional como a composição florística de diferentes

Os estudos realizados por diferentes autores (Guarim Neto, 1978; 1985; Ferrucci, 1983; 1985; 1996; Acevedo-Rodriguez, 1987; 1989; 1993; Ferrucci \& Acevedo-Rodriguez, 1997) mostram a importância de membros da família Sapindaceae, considerando a flora de diversas regiões. Nesse contexto, é oportuno ressaltar as contribuições de Guarim Neto (1979; 1983; 1983a; 1992; 1993; 1993a; 1994;
$1995 ; 1996$; 1996a), com estudos referentes à morfologia e taxonomia da família Sapindaceae, contemplando os gêneros Talisia e Cupania, em nível de revisões taxonômicas, enfocando a ocorrência em áreas brasileiras como a Amazônia, o Centro-Oeste (Estados de Mato Grosso, Goiás, Mato Grosso do Sul), o Sudeste e o Sul, incluindo a ocorrência e distribuição da família em áreas extra-brasileiras.

Tratando de estudos sobre os usos de espécies de Sapindaceae, Guarim Neto (1992) mostra dados referentes à Cupania vernalis Camb.; Guarim Neto et al. (1999/2000), referem-se à Sapindus saponaria L. e Guarim Neto et al. (2000), considerando a etnobotânica de diferentes espécies.

Portanto, este trabalho foi realizado com objetivo de contribuir para o conhecimento, valorização e divulgação de Talisia esculenta (A. St.-Hil.) Radlk., denominada comumente de "pitomba" ou "pitombeira", uma vez que é uma

${ }^{1}$ Professor Titular do Departamento de Botânica e Ecologia, Instituto de Biociências, Universidade Federal de Mato Grosso. CEP 78060-900 - Cuiabá - MT. e-mail: gguarim@terra.com.br

${ }^{2}$ Bióloga. Mestranda em Saúde e Ambiente, Área de Etnobotânica. ISC/UFMT. Bolsista CNPq.

${ }^{3}$ Professora da Universidade Federal da Paraíba. Doutoranda em Saúde e Ambiente, Área de Etnobotânica. ISC/UFMT. Bolsista CAPES. 
espécie de valor econômico tradicional e distribuída por diferentes regiões brasileiras.

\section{MATERIAL E MÉTODOS}

A metodologia utilizada para este estudo baseia-se nas técnicas indicadas para um estudo botânico formal (Guarim Neto 1978; 1985; Acevedo-Rodriguez, 1989; 1993), utilizando as exsicatas da espécie, envolvendo os nomes vulgares, usos, ocorrência e distribuição, bem como aspectos da metodologia empregada por Motley (1994) em seus estudos na família Araceae, que indica inicialmente uma abordagem sobre os dados já obtidos sobre a planta em estudo, assim como complementa com outros, direcionados para o repertório geral e de uso das espécies.

$\mathrm{O}$ estudo foi desenvolvido em material herborizado do acervo dos herbários abaixo relacionados, consultados pelo primeiro autor, quando da revisão taxonômica de Talisia Aublet (Guarim Neto, 1978):

A - Arnold Arboretum, Harvard University, Estados Unidos da América do Norte;

BM - British Museum (Natural History), Inglaterra;

BR - Jardin Botanique National de Belgique, Bélgica;

C - Botanical Museum and Herbarium, Dinamarca;

F - Field Museum of Natural History, Estados Unidos da América do Norte;

$\mathrm{G}$ - Conservatoire et Jardin Botaniques, Suiça;

GH - Gray Herbarium of Harvard University, Estados Unidos da América do Norte;

IAC - Instituto Agronômico do Estado (Phanerogamic Herbarium), São Paulo, Campinas), Brasil; Brasil;

IAN - Instituto Agronômico do Norte,

INPA - Instituto Nacional de Pesquisas da Amazônia, Brasil;

IPA - Instituto de Pesquisas Agronômicas, Brasil;

K - Royal Botanic Gardens, Inglaterra;

L - Rijksherbarium, Holanda;

M - Botanische Staatssammlung, Alemanha;

MG - Museu Paraense Emilio Goeldi, Brasil;
MICH - Herbarium of the University of Michigam; Estados Unidos da América do Norte;

MO - Herbarium of Missouri Botanical Garden, Estados Unidos da América do Norte;

NY - The New York Botanical Garden, Estados Unidos da América do Norte;

R - Museu Nacional do Rio de Janeiro, Brasil;

RB - Jardim Botânico do Rio de Janeiro, Brasil;

S - Swedish Museum of Natural History (Naturhistoriska riksmuseet), Suécia;

UC - Herbarium of the University California, Estados Unidos da América do Norte;

UFMT - Herbário da Universidade Federal de Mato Grosso, Cuiabá, Mato Grosso, Brasil;

US - U. S. National Herbarium, Departament of Botany, Smithsonian Institution, Estados Unidos da América do Norte;

W - Naturhistorisches Museum, Áustria, citados após a descrição botânica da espécie, bem como acrescidos de dados obtidos em trabalhos de campo a partir da revisão, especialmente em território nacional. As estruturações taxonômicas seguem as proposições de Radlkofer (1900) e Cronquist (1981).

\section{RESULTADOS}

Descrição taxonômica atualizada da espécie

Talisia esculenta (A. St.-Hil.) Radlk. in Sitzb. Math.-Phys. Akad. Münch. 8 (5): 345. 1878; in Engler \& Prantl., Nat. Pflanzenf. 3(5): 3324, fig. 166E-H. 1895; in Martius, Fl. Bras. 13(3): 537-539. t.111. 1900; in Engler \& Diels, Das Pfanzenr. 4(165): 837-838. 1921; Barkley in Lilloa 38(14): 168. 1957; Guarim Neto, Diss. de Mestrado, 256p. 1978.

TYPUS: Saint-Hilaire s/n. Brasil. Minas Gerais, Sertão do Rio São Francisco, fl, set. 1816-21 (B, provavelmente destruído; DC; P: non vidi.)

Árvores de 5-15m de altura (raramente arbustos), muito frondosas; fuste cilíndrico, estriado, acinzentado ou escurecido, lenticeloso; ramos cilíndricos, estriados, tenuemente puberulentos. Folhas pari- (impari-) penadas; 
pecíolo de $3-10 \mathrm{~cm}$ de comprimento, cilíndrico, profundamente estriado, pubescente, escurecido, ráquis também cilíndrica, estriada (algumas vezes angulosa), pubescente, escurecida; peciólulo de $1-5 \mathrm{~mm}$ de comprimento, subcaloso, com pilosidade amarelada e algumas vezes piloso-seríceo; 2-4 pares de folíolos, $4-6 \mathrm{~cm}$ de comprimento por $2,5-7 \mathrm{~cm}$ de largura, exatamente opostos ou alternos, desde ovados ou oblongo-lanceolados até pouco obtusos ou obtuso-acuminados, base assimétrica, membranáceos, até 13 pares de nervuras laterais, nervura principal ligeiramente proeminente na face superior, proeminente na inferior, muito brilhantes na face superior, com pêlos esparsos na inferior. Inflorescência em tirsos, até $20 \mathrm{~cm}$ de comprimento por $3-6 \mathrm{~cm}$ de largura, terminais, axilares, constituídos de dicásios curto-estipetados. Flores alvas, alvoamareladas, $5-8 \mathrm{~mm}$ de comprimento, muito aromáticas; pedicelo até $4 \mathrm{~mm}$ de comprimento, bracteolado; bractéolas lanceoladas, pubescentes. Cálice partido até quase a base, sépalas ovadas, ligeiramente obtusas, puberulentas externa e internamente, esparsamente cilioladas nas margens. Pétalas com quase o dobro do comprimento do cálice, estreitamente lanceoladas, base externamente ciliado-barbada, internamente glabra, curtounguiculadas, unha vilosíssima. Escamas nitidamente bífidas, estreitamente lanceoladas, densamente vilosas, liguladas, lígulas intumescidas, glabérrimas. Disco sinuoso, intumescido, glabro. Estames filiformes e pilosos, anteras oblongas, apiculadas. Ovário ovóide, densamente piloso, estilete curto, estigma globoso. Frutos jovens até $1 \mathrm{~cm}$ de comprimento, quase ovóides, apiculados, hirtelóides, amarelados, com resíduos do cálice e dos estames, os maduros até $2,5 \mathrm{~cm}$ de comprimento, quase globosos, granulados, apiculados e pouco pubescentes, também pulverulentos, amarelados e com resíduos do cálice, geralmente monospérmicos, algumas vezes abortivos. Sementes alongadas, testa avermelhada in vivo, escura quando seca, envolvida por um arilo róseo-esbranquiçado, comestível, cotilédones espessos, quase iguais, superpostos.

Material examinado: BRASIL: Amazonas: Corner 57 fl (IAN); G. Guarim
Neto $79 \mathrm{fl}$ fr (INPA); $80 \mathrm{fl}$ (INPA); F. Mello s/ $n$ fl (INPA 2275; MG 4184); R.Vilhena $11 \mathrm{fl}$ $\&$ fr (INPA); Col. não identificado $s / n$ est (INPA 8617). Pará: G. A Black 15397 fl (IAN); P. Cavalcante et al. $1675 \mathrm{fr}(\mathrm{MG}) ;$ A Macedo 4048 fl (MO; RB; S: US); Pessoal do Museu $s / n$ fl (INPA 12;607;MG 9743; UB). Paraná: G.Hatschbach 14295 fl (NY). Maranhão: $A$ Ducke $s / n$ fl (MG 424); R. L. Froés 34925 fr (IAN). Ceará: H. M. Curran 30 est (GH; US); A Ducke s/n fl (MG 1634); Eugênio $796 \mathrm{fl}$ (RB); G. Gardner 1501 fl (BM; G; GH; K; NY; S; W); E. Ule 9064 fl (K; L; M; US). Paraíba: O Diogenes $s / n$ fl ( 36484); J. M. Vasconcelos $s / n$ fl (IAC 7735); P. von Luetzlburg $s / n \mathrm{fl}$ (M)). Pernambuco: $G$. Gardner 951 fl (BM; F; G; GH; K; NY; S; W); $954 \mathrm{fl}$ (BM); D. B. Pickel 146 fl (BM; F); 306 fl (GH; IAN; MICH; US); IPA 6801 fl (IPA); $G$. A Ramage $s / n \mathrm{fl}$ (BM); V. Sobrinho $s / n \mathrm{fl}$ (R 112199); INPA 68881 fl (INPA); IPA $330 \mathrm{fl}$ (IPA); $R B 93921$ fl (RB). Bahia: G. A Black $17714 \mathrm{fr}$ (IAN); J. Martin $s / n \mathrm{fl}$ (L); J. M. Pires $58132 \mathrm{fl}$ (MO); Zehtner 469 fr (M; R). Minas Gerais: M. Barreto $6084 \mathrm{fl}$ ( R ); M Claussen $s / n \mathrm{fl}(\mathrm{F} ; \mathrm{G} ; \mathrm{GH} 0 ; 500 \mathrm{fl}(\mathrm{NY}) ; 517 \mathrm{fl}(\mathrm{C} ; \mathrm{CH}$; NY; S); 2537 fl (L); 20862 fl (C; G; R; S); $L$. Netto $s / n$ fl (BR); G. M. Nunes $72 \mathrm{fl} \& \mathrm{fr}(\mathrm{RB})$; J. E. Oliveira $s / n$ fr (IAN 14902); IAN 15952 fl (IAN); L. Riedel 2645 fl (NY; US0. Rio de Janeiro: E. A Botelho $s / n$ est (RB 102191); Glaziou 801 est (BR; C); $15447 \mathrm{fl}$ (G); J. G. Kuhlmann $s / n$ fl (RB 137579); P. Ochioni $s / n$ fl (INPA16241; RB 20898). Mato Grosso: $F$. C. Hoehne $4507 \mathrm{fl}(\mathrm{M}) ; 4508 \mathrm{fl}$ ( R ); J. G. Kuhlmann $922 \mathrm{fl}$ ( R ); $923 \mathrm{fl}$ ( R ); $924 \mathrm{fl}$ ( R ); $925 \mathrm{fl}$ ( R ); G. Malme $2300 \mathrm{fl}(\mathrm{GH} ; \mathrm{R} ; \mathrm{S}$; US); P . S. Manso $s / n$ fl (BR; G; K; M; NY; W); G. T. Prance et al. $59317 \mathrm{fl}(\mathrm{M} ; \mathrm{MO} ; \mathrm{S})$; L. Riedel 1170 fl (A; NY; US). Goiás: $O H$. Leonardos $s / n$ fl (RB 70261). BOLÍVIA: $T$. Herzog 1258 fr (L; M; S) : H. H. Rusby 1390 fr (F; MICH; NY; US); E. Schmidt 111 fl (M); J. Steinbach 6595 fl (A; BM; F; G; K; S); 7604 fl (A; BM; F; GH; K; M; MO; NY; S). PARAGUAI: K. Fiebrig 4178 fl (BM; G; GH; K; L; M); 4683 fl (L); E. Hassler $1454 \mathrm{fl}(\mathrm{G})$; 4896 fl (BM; G; NY; UC); 7413 fl \& fr (A; BM; C: K; MICH; MO; NY; RB; S; UC; US; $\mathrm{W}) ; 7413^{a}$ fl (A; BM; C; G; MICH; MO; S; UC; W); 10903 fr (M); E. Rojas s/n fl (G). 
Local não identificado: G. Gardner $1051 \mathrm{fl}$ (W); A Glaziou $6491 \mathrm{fl}$ ( C ); $8309 \mathrm{fl}$ (C; K); $10424 \mathrm{fl}(\mathrm{C} ; \mathrm{K}) ; 10559$ est ( C ); E. P. Heringer et al. $9847 \mathrm{fl}(\mathrm{RB})$.

Nomes vulgares: BRASIL: Amazonas, Pará, Maranhão, Ceará, Paraíba, Bahia, Minas Gerais, Rio de Janeiro, Mato Grosso e Goiás: pitomba, pitombeira. Pernambuco: Pitombeira, pitombarana. Bolívia: Pitón, pitón-dulce. Paraguai: Carayá bóla, Carayá-vola (Cavalcante, 1991). Segundo Silva et al. (1977), recebe ainda no Brasil o nome popular de olhode-boi. Vale salientar que no Brasil, os nomes populares mais comuns para esta espécie são pitomba e pitombeira.

Distribuição geográfica e tipos vegetacionais. Habita as matas de terra firme ao redor de Manaus (AM), assim como as capoeiras ralas do Amazonas, Pará, Maranhão, Ceará, Paraíba, Bahia, Minas Gerais, Rio de Janeiro, Pernambuco e a mata pluvial do Paraná (Guarim Neto, 1978). Também adaptada aos cerrados e cerradões de Mato Grosso e Goiás. Esta espécie é encontrada por quase todo o Brasil em estado nativo, silvestre ou em cultivo. Sua distribuição atinge até a Bolívia e o Paraguai. Segundo Cavalcante (1991), a pitombeira é originária da parte ocidental da Amazônia onde provavelmente ainda ocorre no estado silvestre. Entretanto, o material-tipo da espécie foi coletado no sertão do rio São Francisco como descrito por Saint-Hilaire (1828) e apresentado por Guarim Neto (1978). Atualmente, a espécie encontra-se cultivada ou propagada espontaneamente em quase todo o território brasileiro, desde o Amazonas até o Rio de Janeiro e também no Paraguai e na Bolívia. No Pará, é pouco cultivada e, conseqüentemente, os frutos não são comumente encontrados nas feiras (Cavalcante, 1991). Todavia, é muito comum no Amazonas, especialmente na capital, Manaus e arredores, onde é cultivada nos pomares domésticos, pelos frutos e para sombreamento. Segundo Silva et al. (1994), ocorre em mata calcária e cerradão. Segundo Dubs (1998), no estado de Mato Grosso do Sul, Talisia esculenta ocorre na Fazenda Salina (pantanal), no município de Aquidauana, e em Mato Grosso, na Fazenda Currupira, em Cáceres e em Cuiabá. As observações de campo efetuadas comprovam o cultivo da espécie em quintais urbanos e rurais em Mato Grosso, e na capital, Cuiabá, pode ser encontrada com certa freqüência arborizando áreas domésticas. Segundo Lorenzi (1992), ocorre desde o Amazonas, Pará e Maranhão até o Rio de Janeiro, nas florestas pluviais amazônica e atlântica; é particularmente freqüente na Amazônia ocidental e no norte do Espírito Santo (Vale do Rio Doce), encontrada tanto no interior da mata primária densa como em formações secundárias, porém sempre em várzeas aluviais e fundo de vale.

Aspectos fenológicos e germinação. Produz anualmente moderada quantidade de sementes viáveis (Lorenzi, 1992). Floresce durante os meses de agosto-outubro e a maturação dos frutos ocorre a partir daí, sendo mais pronunciada nos meses de janeiro-março, dependendo da região. Segundo Barbosa (1972), a germinação é hipógea, cujos cotilédones não são elevados acima do solo, permanecendo parcialmente enterrados no substrato.

Usos. São usados tanto os frutos como as cascas, folhas e a madeira. Segundo Lorenzi (1992), a madeira é empregada para obras internas na construção civil, como forros, molduras, batentes, tábuas para assoalho, para carpintaria e caixotaria. Os frutos são comestíveis (Silva et al. 1994), comercializados nas feiras da região norte e nordeste do país; muito procurados também por pássaros. A árvore é amplamente cultivada em pomares domésticos de todo país. É indicada para o plantio em áreas degradadas de preservação permanente. Segundo Corrêa (1974), as cascas e folhas contêm tanino e a seiva é ictiotóxica. Em Manaus, os frutos comestíveis são vendidos nas estradas, nas esquinas e ruas do centro comercial assim como nas feiras públicas (observação pessoal), onde são amarrados vários "cachos", constituindo uma unidade. Esta mesma forma de comercialização é também observada em Fortaleza, Terezina e Cuiabá. No mercado do Ver-O-Peso (Belém), foi observado o comércio dos frutos, especialmente do final para o início do ano subseqüente. Ainda em Manaus, uma prática interessante é a de introduzir as inflorescências ainda na árvore, em um recipiente de vidro, até que os frutos se desenvolvam. Ato contínuo, corta-se pela parte 
que sustenta os frutos, com estes no interior do vidro, coloca-se aguardente de boa qualidade, veda-se o vidro e "deixa curtir". Assim, tem-se um licor, conhecido por cajuína. Segundo Prance \& Silva (1975), as sementes são tidas como antidiarréicas e usadas como adstringentes. Segundo Guarim Neto (1987; 1996), o chá das sementes é utilizado para amenizar os problemas de desidratação. Por outro lado, o chá das folhas é indicado para as "dores de cadeira" e para os problemas renais.

\section{BIBLIOGRAFIA CITADA}

Acevedo-Rodriguez, P. 1987. Two new species of Serjania (Sapindaceae) from Brazil. Brittonia, 39:348-352.

Acevedo-Rodriguez, P. 1989. The systematics of Serjania section Platycoccus (Sapindaceae). PhD. Thesis, The City University of New York, New York, 271p.

Acevedo-Rodriguez, P. 1993. Systematics of Serjania (Sapindaceae). Part 1: A revision of Serjania Sect. Platycoccus. Mem. New York Bot. Gard., 67:1-93.

Barbosa, D. C. de A. 1972. Fruto, germinação e crescimento de plântulas de Talisia esculenta Radlk. (Sapindaceae). Bot. Estud. e Pesq., 3(4):1-8.

Cavalcante, P. B. 1991. Frutos comestíveis da Amazônia. Belém, Museu Paraense Emílio Goeldi. 279 p.

Corrêa, P. M. 1974. Dicionário das plantas úteis do Brasil e das exóticas cultivadas. Rio de Janeiro, Ministério da Agricultura/IBDF. Vol. V. p. 513-514.

Cronquist, A. 1981. An integrated system of classification of flowering plants. New York, Columbia University Press. 1262p.

Dubs, B. 1998. Prodromus Florae Matogrossensis. Parte I. Checklist of Angiosperms, Parte II. Types from Mato Grosso. p.221-230.

Ferrucci, M. S. 1983. Novedades en Serjania (Sapindaceae). Bonplandia, 5(26):143-146.

Ferrucci, M. S. 1985. Novedades en Sapindaceae-Eupaullinieae de Brasil. Bol. Soc. Argent. Bot., 24(1/2):107-124.

Ferrucci, M. S. 1996. Urvillea paucidentata y
Serjania adenophylla (Sapindaceae), dos especies nuevas de Mato Grosso, Brasil. Bonplandia, 9(1/2):19-23.

Ferrucci, M. S. \& Acevedo-Rodriguez, P. 1997. New and noteworthy species in the Paullinieae tribe (Sapindaceae). Brittonia, 49(4):441-448.

Guarim Neto, G. 1978. Revisão taxonômica das espécies brasileiras do gênero Talisia Aublet (Sapindaceae). Dissertação de Mestrado, INPA/FUA, Manaus, 256p.

Guarim Neto, G. 1979. Estudos em Sapindaceae I. Novas espécies de Talisia Aublet para o Brasil. Acta Amazonica, 9 (2):233-239.

Guarim Neto, G. 1983. Estudos em Sapindaceae V. Contribuição à sistemática de Talisia olivaeformis (Kunth) Radlk. Caldasia, 13 (65):701-708.

Guarim Neto, G. 1983a. Talisia mollis Kunth var. marleneana G. Guarim Neto, var nov. (Sapindaceae). Acta Amazonica, 13 (34):497-500.

Guarim Neto, G. 1985. Estudos taxonômicos em Cupania L. (Sapindaceae): as espécies brasileiras. Tese de Doutorado, INPA/ FUA, Manaus, 272p.

Guarim Neto, G. 1987. Plantas utilizadas na medicina popular do estado de Mato Grosso. Brasília, MCT/CNPq. 58p.

Guarim Neto, G. 1992. Aspectos taxonômicos e fitogeográficos de Cupania vernalis Camb. (Sapindaceae). Bol. Inst. Biociênc., 1:11-23.

Guarim Neto G. 1993. Novas espécies de Cupania L. (Sapindaceae) para o Brasil. Eugeniana, 20:7-15.

Guarim Neto, G. 1993a. Sapindaceae Jussieu. Relatório parcial II. Cuiabá, UFMT/ CNPq. 70p.

Guarim Neto, G. 1994. Flora dos Estados de Goiás e Tocantins - Coleção Rizzo: Sapindaceae. Goiânia, Editora UFG. 61p.

Guarim Neto, G. 1995. A família Sapindaceae para a flora do Estado de Mato Grosso do Sul, Brasil. Relatório de pesquisa. Cuiabá, UFMT/CNPq. 41p.

Guarim Neto, G. 1996. Plantas medicinais do 
Estado de Mato Grosso. Brasília, ABEAS. $72 \mathrm{p}$.

Guarim Neto, G. 1996a. Ocorrência e distribuição da família Sapindaceae Jussieu nos Estados de Mato Grosso, Goiás e Tocantins. Bol Mus. Par. Emílio Goeldi, 12(2):227-236.

Guarim Neto, G.; Santana, S. R. \& Silva, J. V. da. 2000. Notas etnobotânicas de Sapindaceae Jussieu. Acta bot. bras., 14(3):327-334.

Guarim Neto, G.; Silva, J. V. da \& Santana, S. R. 1999/2000. Sapindus saponaria L. (Sapindaceae): notas botânicas. Eugeniana, 24:7-13.

Lawrence, G. H. M. 1951. Taxonomy of vascular plants. New York, The Mac-Millan Company. 823p.
Lorenzi, H. 1992. Árvores brasileiras. Nova Odessa, Ed. Plantarum. p. 315-322.

Motley, T. J. 1994. The ethnobotany of sweet flag, Acorus calamus (Araceae). Economic Botany, 48 (4): 397-412.

Prance, G. T. \& Silva, M. F. 1975. Árvores de Manaus. Manaus, CNPq/INPA. 312p.

Radlkofer, L. 1900. Sapindaceae. In: Martius, C. F. - Flora Brasiliensis. 13 (3):226-680.

Saint-Hilaire, A. 1828. Plantes usuelles des brésiliens. France. 14(68):1-4.

Silva, M. F. da.; Lisboa, P. L. B. \& Lisboa, R. C. L. 1977. Nomes vulgares de plantas amazônicas. Manaus, INPA/CNPq. 222 p.

Silva, J. A.; Silva, D. B.; Junqueira, N. T. V. \& Andrade, L. R. M. 1994. Frutas nativas do cerrado. Planaltina, EMBRAPA/CPAC. $166 \mathrm{p}$. 